\title{
KATHRIN KOLLMEIER
}

\section{»HINAUS MIT ALLEN STÖRENFRIEDEN!« DER DISZIPLINARRECHTLICHE AUSSCHLUSS AUS DER HITLER-JUGEND ALS AUSGRENZUNG AUS DER NS-VOLKSGEMEINSCHAFT}

Mit dem Schlachtruf "Hinaus mit allen Störenfrieden!« warb die nationalsozialistische Jugendorganisation »Hitler-Jugend " 1935 um die Teilnahme Jugendlicher. Gemeinsam mit der Forderung nach »Einheit der Jugend in der Hitler-Jugend " ${ }^{1}$ bringt ein regionales Werbeplakat aus der Zeit des Ausbaus der Parteinachwuchsorganisation zum monopolisierten Jugendverband und damit zur zentralen Sozialisationsinstanz im NS-Staat eine Doppelfigur auf den Punkt: Das Zusammenspiel von Strategien der Integration und der Ausgrenzung verbindet sich in der zentralen Herrschaftstechnik der "Volksgemeinschaft «, die - ideologisch konzipiert, propagandistisch inszeniert und sozialpolitisch praktiziert - hier als eine Figuration der deutschen Diktatur betrachtet wird. Das Interesse liegt dabei auf der kleinteiligen Umsetzung dieser Technik am Beispiel der Disziplinarpolitik des monopolisierten Jugendverbandes, der die doppelte Struktur einer "Gliederung " der NSDAP und zugleich einer staatlichen Institution aufwies ${ }^{2}$.

Traditionell als Mittel der Mobilisierung und Integration verstanden, bildet die nationalsozialistische Jugendorganisation zugleich auch den Ort, an dem die gesellschaftliche Ein- und Ausgrenzung für Kinder und Jugendliche beider Geschlechter im Alter von 10 bis 18 Jahren vollzogen wurde. Der Blick auf die genuin nationalsozialistische Organisation, die seit der sogenannten »Kampfzeit» der NSDAP wesentliche Entwicklungsschritte mit der Partei teilte, ermöglicht mit der Untersuchung der Prozesse dieser Abgrenzung zugleich, die lange Zeit forschungsprägende methodische Trennung zwischen Repräsen-

\footnotetext{
' Plakat der Gaupropagandaleitung Baden, Hitler-Jugend. Bundesarchiv (BA) Koblenz, Plakatesammlung, 3/11/42.

${ }^{2}$ Die Untersuchung gehört zu meinem Dissertationsprojekt »Gesellschaftliche Ausgrenzung und disziplinarische Ordnung im Nationalsozialismus. Die Disziplinarpolitik der HitlerJugend « und bezieht sich auf die Gesamtorganisation, die, sofern nicht anders gekennzeichnet, hier unter »Hitler-Jugend« verstanden wird.
} 
tanten des NS-Systems auf der einen und ihren Opfern auf der anderen Seite $\mathrm{zu}$ überwinden ${ }^{3}$.

Als »klassenunspezifischer, aber allumfassender Akteur « bieten sich die Altersgruppe der Jugendlichen und die ihnen gewidmete Organisation als Untersuchungsgegenstand aus mehreren Gründen an. Bekanntermaßen erfuhr "Jugend" als gesellschaftlich umfassende Gruppe mit hohem Symbolwert eine intensive politische Aufladung, auf die sich die NSDAP und namentlich die Hitler-Jugend selbst beriefen; Selbstdarstellungen und Propaganda strapazierten den Topos von der Jugendlichkeit der gesamten nationalsozialistischen "Bewegung « und des Regimes ${ }^{5}$. Dem zudem mit dem Namen des »Führers" hervorgehobenen Verband wurde dadurch eine zentrale Bedeutung innerhalb der "Bewegung" und für ihre dauerhafte Durchsetzung zugewiesen. Als Potential der Partei und Garant ihrer Zukunftsfähigkeit verhieß »Jugend« für die politische Utopie einer nationalsozialistischen Gesellschaft als "Volksgemeinschaft« die größtmögliche Ausstrahlung der eingeübten neuen Gesellschaftsordnung. Daher mußte, so die Hypothese, eine Kindern und Jugendlichen gewidmete Organisation für Gefährdungen oder Einschränkungen der Ordnung besonders sensibel sein.

Tatsächlich hatte die Hitler-Jugend unter den Parteiorganisationen die am weitesten gespannte Zielgruppe, die sie mit einem Erfassungsgrad von 87\% der Jugend beider Geschlechter zu Beginn des Jahres 1939 auch weitgehend erreichte $^{6}$. Eine weitere Besonderheit im Vergleich zu den freiwilligen Gliederungen der "Sturm-Abteilung" (SA) und der "Schutz-Staffel" (SS) war die ab 1939 bestehende gesetzliche Verpflichtung für nicht-jüdische deutsche Kinder und Jugendliche zur Mitgliedschaft, wobei allerdings der Eindruck der Pflichtmitgliedschaft der späten Regelung lange vorausgriff ${ }^{7}$. Als Nachwuchsorgani-

${ }^{3}$ So die zurecht kritisierte Hauptperspektive der umfangreichen Forschung zur Opposition Jugendlicher im Nationalsozialismus. Vgl. Wilfried BREYVOGEL, Thomas STUCKERT, Kommentierte Bibliographie zum Jugendwiderstand im Nationalsozialismus, in: Wilfried BREYVOGEL (Hg.), Piraten, Swings und Junge Garde. Jugendwiderstand im Nationalsozialismus, Bonn 1991, S. 326-351, S. 328.

${ }^{4}$ Heinz BUDE, Das Altern einer Generation. Die Jahrgänge 1938 bis 1948, Frankfurt a. M. 1995, S. 26.

${ }^{5}$ Exemplarisch für die Selbstdarstellungen der Hitler-Jugend: Baldur von SCHIRACH, Die Hitler-Jugend. Idee und Gestalt. Leipzig o.J. [1936, zuerst 1934], S. 180; für die Mitgliederund Wählerstruktur der NSDAP und Motivationen vor allem vor 1933 und in den ersten Jahren des Nationalsozialismus wurde eine relative Jugendlichkeit nachgewiesen, vgl. Michael H. KATER, Generationskonflikt als Entwicklungsfaktor in der NS-Bewegung vor 1933, in: Geschichte und Gesellschaft 11 (1985) S. 217-243; DERS., The Nazi Party. A Social Profile of Members and Leaders, Oxford 1983, S. 139, 141-145.

${ }^{6}$ Karl-Heinz JAHNKE, Michael BudDRUS (Hg.), Deutsche Jugend 1933-1945. Eine Dokumentation, Hamburg 1989, S. 15.

${ }^{7}$ Erst die beiden am 25.3.1939 erlassenen Durchführungsverordnungen zum "Gesetz über die Hitler-Jugend « vom 1.12.1936 (Reichsgesetzblatt 1936, Teil I, S. 993; Reichsgesetzblatt (RGBI). 1939, Teil I, S. 709-710) bildeten eine gesetzliche Grundlage für die Verpflichtung 
sation versorgte die Hitler-Jugend mit ihren nach Altersklassen und Geschlecht differenzierten Unterorganisationen nicht nur die Partei, sondern sämtliche Gliederungen und weitere Parteiverbände und entwickelte sich so zu einer der wichtigsten der zahllosen Massenorganisationen von NS-Staat und -Gesellschaft, in deren dichtem Netz - eng miteinander verschränkt und aufeinander bezogen - nicht nur Strategien der Mobilisierung vollzogen wurden, sondern auch die Mechanismen der Ausgrenzung. Der »Dienst « in der Hitler-Jugend bildete die Basis dieser neuen Gesellschaftsordnung.

Große Menschenmassen zu bewegen, sie zur Mitarbeit zu mobilisieren, ihr Interesse langfristig zu erhalten und ihren beständigen Einsatz zu organisieren, erfordert einerseits flexible Ordnungssetzungen, andererseits die verbindliche Durchsetzung dieser Ordnungen gegenüber den Mitgliedern. Um diese Aufgaben zu erfüllen, entwickelte die Hitler-Jugend ein eigenes Disziplinarsystem, das sie über den gesamten Herrschaftszeitraum des Nationalsozialismus systematisch ausbaute. Als gesellschaftlicher Ordnungsprozeß mit weitreichenden sozialen und politischen Konsequenzen setzte dieser Disziplinarmechanismus gesellschaftlich umfassend, aber konzeptionell kleinteilig am Einzelnen an. Die Entwicklung dieses Disziplinarsystems und die Interessen der Einflußnahme lassen sich auch als Ausdruck des Verhältnisses der Parteijugendorganisation mit totalitärem Anspruch zur NSDAP und der Beziehung der staatlichen und parteilichen Behörde der "Reichsjugendführung der NSDAP» bzw. der "Jugendführung des Deutschen Reiches« zu anderen staatlichen Stellen im doppelten Organisationsgefüge des NS-Staates verstehen. Hier liegt jedoch das Interesse auf den disziplinarischen Ordnungsprozessen selbst. Mit der Strafe des Ausschlusses konzentriert die Darstellung sich auf deren disziplinarische Kernfunktion. Denn die Analyse dieser paradoxen Figur der strafweisen Entfernung aus einer auf »totale« Erfassung angelegten Organisation ermöglicht, Ordnungskonzepte für eine widersprüchliche und in ihrem umfassenden Erziehungsanspruch utopische Jugendorganisation sichtbar zu machen und damit eine Feinmechanik der nationalsozialistischen Herrschaft zu verdeutlichen.

Erst vor dem Hintergrund des ausgefeilten Disziplinarsystems erschließt sich die Bedeutung der Strafe der Entfernung aus der Hitler-Jugend, des "Ausschlusses«, als gesellschaftliches Ausgrenzungsverfahren: Über die Zugehörigkeit zur "Volksgemeinschaft«, die Partizipation an der NS-Gesellschaft, über berufliche Chancen und Entwicklungsmöglichkeiten, über den Rechtsstatus und Rechtssicherheit wurde auch in dieser genuin nationalsozialistischen Organisation entschieden. Die Techniken des Disziplinarsystems der HitlerJugend gliedern sich dabei in Mittel der dokumentierenden Erfassung, der 
normalisierenden Überwachung und die Strafe des Ausschlusses aus dem Verband, die mit Michel Foucault als Techniken von Gewaltverfahren verstanden werden ${ }^{8}$. Die zentrale Quellengrundlage bildet dabei die »Warnkartei $«$ der Reichsjugendführung, in der alle "unehrenhaften« Ausschlüsse aus der gesamten Jugendorganisation mit ihren Untergliederungen verzeichnet wurden, um reichsweit eine Wiederaufnahme der Ausgeschlossenen zu verhindern. Sie dokumentiert damit die Anwendung der schärfsten - und mit Foucault einzigen - Strafe dieser Parteigliederung gegenüber ihren jugendlichen Mitgliedern, Funktionären und fachlichen Mitarbeitern im Rahmen des eigenen dienstrechtlichen Disziplinar- und Strafsystems. Die Kartei bürgt mit knapp 5000 Einträgen aus der gesamten Herrschaftszeit des Nationalsozialismus für die massenhafte Realität des Verfahrens; ihr Schwerpunkt liegt dabei mit den Jahren 1934-1939 in der Phase der sich etablierenden Ordnung der Parteigliederung als staatlicher Jugendverband. Sammelveröffentlichungen für die anschließenden Zeiträume ergänzen die Vollständigkeit dieser offiziell zum Juli 1939 eingestellten Kartei ${ }^{9}$. Eingebettet in die reichhaltige Drucksachen-Überlieferung der Hitler-Jugend - Verordnungs- und Befehlsblätter, akribisch kommentierte Dienstanweisungen und interne Denkschriften sowie juristische und sozialpolitische Fachzeitschriften und Monographien - und teilweise aus der Sekundärüberlieferung erschlossene Akten der Reichsjugendführung verdeutlichen diese kargen seriellen Quellen die Intentionen, Argumentationen und die Politik der Reichsjugendführung. Auf dieser Materialgrundlage folgt hier nach der Darstellung des Disziplinarsystems und seiner zentralen Disziplinartechniken die Analyse von Veränderungen nach der organisationsgeschichtlichen und politischen Zäsur von 1939. Abschließend wird das zugrunde liegende Gesellschaftsbild aus dem dichotomischen Disziplinarkonzept mit den beiden Polen »Erziehen« und »Warnen« skizziert.

\footnotetext{
${ }^{8}$ Michel FouCAULT, Surveiller et punir. Naissance de la prison, Paris 1999 [1975].

9 BA Berlin, Berlin Documènt Center (BDC). Der Bestand umfaßt ca. 11000 Karten mit 4779 Fällen, die stark formalisierte Informationen zu biographischen Massendaten und den datierten Vermerk eines Ausschlusses unter formelhafter Angabe der Begründung enthalten. Zur Auswertung der »Wamkartei« vgl. bereits meine Magisterarbeit: Kathrin KOLLMEIER, Die Disziplinarpolitik der Hitler-Jugend: Ausschlußverfahren und Ausschlüsse in den Jahren 1933 bis 1944/45. Humboldt-Universität zu Berlin, 2000. 2698 weitere Fälle von Ausschlüssen und dauerhaften "Ausscheidungen « sind dokumentiert in: Reichsbefehl der Reichsjugendführung der NSDAP. Befehle und Mitteilungen für die Führer und Führerinnen der Hitler-Jugend. Sonderdruck 35/41 K, 25.9.1941, S. 1-51; sowie: Ausschlüsse aus der Verwaltung der Hitler-Jugend durch Verfügung des Reichsschatzmeisters, in: Reichsbefehl 7/K (27.10.1939) mit 80 Ausschlüssen, von denen sechs auch im Sonderdruck $35 / 41 \mathrm{~K}$ erfaßt sind. Eine hier angekündigte weitere Sammlung konnte nicht ermittelt werden.
} 


\section{Das Disziplinarsystem der Hitler-Jugend Erfassen, Überwachen und Strafen}

Ab 1933 wurde die Parteinachwuchsorganisation »Hitler-Jugend« mit den Suborganisationen »Jungvolk«, »Bund Deutscher Mädel« und »Jungmädelbund « zielstrebig in einen hierarchisch organisierten Jugendverband mit staatlichem Erziehungsauftrag umgebaut, dessen Struktur sich eng an den Aufbau der NSDAP anlehnte ${ }^{10}$. Der Reichsjugendführer der NSDAP, Baldur von Schirach, erhielt zunächst in Personal-, dann institutioneller Union zugleich als »Jugendführer des Deutschen Reiches« Befehlsgewalt über sämtliche Jugendliche im Reich, also auch über die Nichtmitglieder. Im Dezember 1936 formulierte das »Gesetz über die Hitler-Jugend « zunächst programmatisch einen umfassenden sittlichen, weltanschaulichen und politischen Erziehungsanspruch für alle »arischen« Kinder und Jugendlichen im Alter zwischen 10 und 18 Jahren durch die Organisation". Parallel zu ihrer massiven Mitgliederrekrutierung durch die Ausschaltung und Einverleibung konkurrierender Jugendverbände entwickelte die Jugendführung in den ersten Jahren des Nationalsozialismus auf der Grundlage vereinsrechtlicher Regelungen ein eigenes disziplinarisches Rechtssystem, das von dokumentarischer Erfassung begleitet wurde.

Mit hohem Personalaufwand wurde die Mitgliederverwaltung betrieben, die im Januar 1934 einheitlich reorganisiert wurde. Als Pendant zur Mitgliederkartei entstand zeitgleich eine zweite Personalerfassung ehemaliger Mitglieder auf »Warnkarten«. Auf regionaler und lokaler Ebene wurden diese »Warnfälle«, deren Wiederaufnahme oder Betreuung mit Geldern und Verantwortung nicht erwünscht war, alphabetisch in die Mitgliederkartei einsortiert. Ein Doppel wurde zugleich zentral in der Warnkartei der Reichsjugendführung archiviert. Als weiterer hier gespeicherter Personenkreis galten sogenannte »jugendgefährdende « Personen; insbesondere handele es sich hierbei um »die Homosexuellen und Angehörige früherer staatsfeindlicher Jugendorganisationen«, wie 1938 definiert wurde ${ }^{12}$. Ihre Informationen erhielt die Kartei nicht nur von den Dienststellen der Hitler-Jugend, hier vor allem von der für das Disziplinarwesen zuständigen »HJ-Gerichtsbarkeit«, sondern auch von der Polizei und Instanzen der Jugendpflege. Auskünfte gewährte sie der Zentralkartei der NSDAP, mit der sie systematisch Warnkarten austauschte, und allen

${ }^{10}$ Zur Entwicklung und Durchsetzung der Jugendorganisation vgl. die mittlerweile klassische Gesamtdarstellung von Amo KLÖNNE, Jugend im Dritten Reich. Die Hitler-Jugend und ihre Gegner, Düsseldorf 1982, emeut Köln 2003.

" Gesetz über die Hitler-Jugend, 1.12.1936, RGB1. 1936, Teil I, S. 993, §2.

12 Schreiben der Jugendführung des Deutschen Reiches, Heinz Boldt, an das Reichsministerium der Justiz, zu Händen des Staatsanwaltes Westpfahl, 12.9.1939. BA Berlin R 3001/alt R 22/1177, Bl. 230. 
Gliederungen. Ordnungsgemäß war die Kartei vor jeder Aufnahme eines über 14-jährigen Jugendlichen in eine Parteiorganisation zu konsultieren ${ }^{13}$.

Der vielzitierte »Totalitätsanspruch« des Jugendverbandes, der mit der Erfassung der - analog zur Parteimitgliedschaft - rassisch definierten 'gesamten deutschen Jugend und aller Lebensbereiche dieser Jugend ein doppeltes jugendpolitisches Monopol verfolgte, spiegelt sich in diesem detaillierten Dokumentationsinteresse. Die komplementäre Personalerfassung in Mitgliederkartei und Warnkartei zielte auf eine vollständige karteitechnische Erfassung aller Kinder, Jugendlichen und Jugendführer, die sich auf diese Weise, gemäß den Standards zeitgenössischer Bürotechnik, übersichtlich, ergonomisch und rationell entweder als Mitglieder und Mitarbeiter der Organisation oder als unerwünschtes Gefahrenpotential identifizieren und verwalten ließen.

Die Überwachung Jugendlicher wurde vor dem Hintergrund des wöchentlichen Hitler-Jugend-Dienstes möglich und durch eigene Organe umgesetzt. Seit 1935 verfügte die Organisation mit der »HJ-Gerichtsbarkeit« über eine eigene Judikative und mit dem "HJ-Streifendienst« über eine eigene Überwachungsexekutive. Als lokale Kontrollstreife älterer HJ-Führer beobachtete der Streifendienst das Verhalten und Auftreten zunächst nur von Angehörigen der Hitler-Jugend und des BDM außerhalb des Dienstes; ab 1937 bestand seine Aufgabe darin, »alle politischen und kriminellen Vorgänge innerhalb der deutschen Jugend zu überwachen und bekämpfen«. Schwerpunkte der "Überwachung" lagen dabei auf konfessionellen Aktivitäten, illegal fortbestehenden Gruppen der ehemaligen »Bündischen Jugend" und homosexuellen Handlungen $^{14}$. In der Feststellung disziplinarischer Tatbestände und als Ermittlungsorgan in den dienstrechtlichen Voruntersuchungen wirkte der HJ-Streifendienst in enger Zusammenarbeit mit der Polizei - vorrangig Gestapo und Sicherheitsdienst - wesentlich an den Disziplinarverfahren mit. Seine Meldungen dienten als empirische Grundlage disziplinarpolitischer Entscheidungen der Jugendführung. Diese umfangreiche Dokumentation ihrer Aktivitäten in monatlichen Berichten und Statistiken sowie regelmäßige Vermißtenmeldungen und "Warnungen« vor polizeilich gesuchten oder unliebsamen Personen ergänzten die Disziplinargewalt. Der als SS-Nachwuchsorganisation elitär konzipierte HJ-Streifendienst kontrollierte auch die weiblichen Untergliederungen, wurde aber zur Zurückhaltung gegenüber den Mädchen und jungen Frauen verpflichtet ${ }^{15}$. Ein temporärer Plan unmittelbar vor Kriegsbeginn, eine Son-

\footnotetext{
${ }^{13}$ Verordnungsblatt der Reichsjugendführung (Hitler-Jugend) III/28, 8.8.1935, S. 3.

14 Verfügung des Stabsführers im "streng vertraulichen“ Reichsbefehl 31/II, 20.8.1937, S. 826. Vgl. zum HJ-Streifendienst auch Armin NOLZEN, Der Streifendienst der Hitlerjugend und die "Überwachung der Jugend«, 1934-1945, in: Christian GeRLaCH (Hg.), Durchschnittstäter. Handeln und Motivation, Berlin 2000, S. 13-51; Gerhard REMPEL, Hitler's children. The Hitler Youth and the SS. Chapel Hill, London 1989, S. 47-106.

${ }^{15}$ Richtlinien für das Einschreiten des HJ-Streifendienstes in besonderen Fällen, in: Vorschriftenhandbuch der Hitler-Jugend. 1. Ausgabe vom 1.1.1942. Bd. II, S. 926.
} 
derformation "BDM-Einsatzdienst« aufzubauen, wurde noch in der Umsetzung abgebrochen ${ }^{16}$. Damit fehlte ein vergleichbarer organisatorischer Überwachungsapparat, der disziplinarische Auffälligkeiten außerhalb der Dienststunden feststellte und meldete, dauerhaft in den Mädchenorganisationen.

Im Rahmen fortlaufend modifizierter Disziplinar- und Strafordnungstexte verwandelte die Reichsjugendführung das Vereinsrecht, Mitglieder und Funktionäre auszuschließen, zu einer zielgerichteten Disziplinarstrafe. Die erste "Disziplinarordnung", der jedes Mitglied mit der Aufnahme in die Organisation unterstand, formulierte im Dezember 1936 die Grundzüge des Disziplinarsystems, das trotz verschiedener Neuregelungen im Detail und mehrerer Umbenennungen in der Substanz bis 1945 stabil blieb ${ }^{17}$. Diese beinhalteten die Ausübung einer vom Reichsjugendführer verliehenen Disziplinarbefugnis durch HJ-Führer, BDM-Führerinnen und eigene "HJ-Richter" gegenüber den Mitgliedern, nach Dienstrang gestaffelte Kompetenzen und einen Katalog von "Disziplinarmitteln«, "gegen den anzuwenden, der die Pflichten verletzt, die ihm die Zugehörigkeit zur Hitler-Jugend auferlegt « ${ }^{18}$. Die Disziplinarordnungen boten daher gleichermaßen gegenüber den Jugendlichen in den Einheiten wie gegenüber dem Führerkorps »eine rechtliche Grundlage, um alle diejenigen Elemente aus der Jugendbewegung zu entfernen, welche durch Unkameradschaftlichkeit und Mangel an Gemeinschaftsgeist den Bestand der Gemeinschaft selbst gefährden “" tät der jugendlichen Träger des sogenannten, aus der bürgerlichen und später bündischen Jugendbewegung übernommenen "Selbstführungsprinzips ${ }^{20}$ gegenüber ihren kaum jüngeren Einheiten stützen, andererseits etwaige Willkür der jungen Führer und Führerinnen begrenzen und damit nach außen die Selbstdisziplinierungsfähigkeit der Jugendorganisation beweisen.

16 Rundschreiben der Reichsreferentin des BDM, Jutta Rüdiger, 31.8.1939. BA Berlin NS 28/32, B1. 3.

${ }^{17}$ Zuvor galt die "HJ-Dienstordnung«, in: Verordnungsblatt II/5, 7.7.1934, S. 3f;; Die Disziplinarordnung für HJ, DJ, BDM und JM, in: Reichsbefehl, streng vertraulicher Sonderdruck. 14.12.1936, S. 3; Neufassung der Disziplinarordnung. Streng vertraulicher Reichsbefehl Sonderdruck 7/39, 29.3.1939; Die Dienststrafordnung der Hitler-Jugend für die Dauer des Krieges. Reichsbefehl 34/K, 2.4.1940, S. 1-11, S. 2; Dienststrafordnung der Hitler-Jugend für die Dauer des Krieges (Neufassung). Reichsbefehl 23/41 K, 19.5.1941; in der durch Anordnungen des Reichsjugendführers $4 / 42$ und 8/42, 15.1.1942, veränderten Fassung auch in: Vorschriftenhandbuch, Bd. II, S. 997-1005.

${ }^{18}$ Disziplinarordnung 1936, S. 3.

${ }^{19}$ ANONYM, HJ im Rechtsleben der Nation, in: Deutsche Justiz 97 (1935) S. 76 f.

${ }^{20}$ Das Prinzip, daß »Jugend von Jugend gefüht werden müsse«, reduzierte die sogenannte »Hohe-Meißner-Formel« der bürgerlichen Jugendbewegung auf die Führungsrolle Jugendlicher innerhalb der Jugendgemeinschaft und diente so als institutionelles Legitimationsprinzip. Vgl. Hermann GIESECKE, Vom Wandervogel bis zur Hitlerjugend. Jugendarbeit zwischen Politik und Pädagogik, Weinheim 1981, S. 22. 
Nach Maßgabe dieser selbst erlassenen Disziplinar- und Strafordnungen übte die Hitler-Jugend als öffentliche Erziehungsgewalt seit 1936 Disziplinarrechte aus, die ihrem Selbstverständnis nach »erzieherisch« wirken sollten, um "Jugendliche, die sich gegen die Ehrauffassung der deutschen Jugend vergangen haben, zur Ordnung zu rufen und mit Hilfe jugendgemäßer Dienststrafen $\mathrm{zu}$ einwandfreier Haltung [...] erziehen ${ }^{21}$. $"$ Erziehung " bedeutete in diesem Sinne jedoch lediglich eine Korrektur von Abweichungen und eine Gewöhnung und Anpassung an die Ordnungsgrundsätze der Hitler-Jugend und entspricht damit Foucaults Beschreibung einer normierenden Disziplinierung.

Die Disziplinierung Einzelner verstand sich dabei zugleich als Erziehung der Gemeinschaft, die »weniger aburteilen und ausstoßen als die Ausrichtung und Zurechtrückung sperriger oder schwieriger Fälle veranlassen und ermöglichen« sollte ${ }^{22}$. Diese »ständige Säuberung« der Hitler-Jugend habe unter ihren Angehörigen wein außerordentlich starkes Gefühl für persönliche Sauberkeit erzeugt«, konstatierte der HJ-Rechtspolitiker Dr. Henry Picker bereits $1935^{23}$. Die »Aufgabe der negativen Auslese« im Rahmen der Disziplinarpolitik wurde als Ergänzung der "positiven Aufgabe der Erziehung und inneren Führung der Jugend« begriffen ${ }^{24}$. Kombinierbare, deutlich am Militär orientierte »Ehrenstrafen « - Mahnungen, Verweise, Degradienungen unterschiedlicher Art oder das Verbot, Ehrenzeichen und bestimmte Uniformteile zu tragen - sollten einen Delinquenten »bei der Ehre packen«. Während des wöchentlichen HJDienstes vollstreckt, waren sie also selbst Teil der »Erziehungsarbeit« der Hitler-Jugend. Sie wirkten normierend und stellten damit einen disziplinierenden Integrationsversuch dar. Nur der »Ehrlose«, dessen Verhalten mit diesen Mitteln nicht mehr korrigierbar erschien, wurde ausgeschlossen:

Es ist das Wesen einer jeden von der Hitler-Jugend angewandten Dienststrafe - mit Ausnahme des Ausschlusses und Ausscheidens - einen bestraften Jugendlichen nicht etwa zu verfemen, sondern erneut in die Gemeinschaft der Jugendlichen einzugliedern. ${ }^{25}$

Der das Disziplinarrecht begründende Ehrbegriff stellte so nicht nur einen Wert der NS-Gemeinschaftsordnung dar, sondern zugleich auch das Prinzip, auf dem die Konstitution und Aufrechterhaltung dieser Ordnung basierte. Er

21 Helmut MÖCKEL, Planmäßige Jugendbetreuung, in: Das Junge Deutschland. Amtliches Mitteilungsblatt des Jugendführers des Deutschen Reiches. Sozialpolitische Zeitschrift der deutschen Jugend 35 (1941) H. 10, S. 241-243, S. 241. Als »Stabsführer« der Reichsjugendführung war Möckel ab 1940 der Stellvertreter des Reichsjugendführers.

${ }^{22}$ Hanns ANDERLAHN, Führung - Erziehung - Strafe, in: Das junge Deutschland 31 (1937) H. 2, S. 52-58, S. 54.

${ }^{23}$ Henry PICKER, Jugendliche Berufskriminalität und ihre Bekämpfung durch die HitlerJugend, in: Das Junge Deutschland 29 (1935) H. 7, S. 292-298, S. 295.

${ }_{24}$ ANONYM, Die neue Disziplinarordnung der HJ, in: Das Junge Deutschland 30 (1936)

H. 12, S. 41 f.

${ }^{25}$ MÖCKEL, Planmäßige Jugendbetreuung, S. 241. 
bezeichnet die Grenze zwischen Disziplinierung und Strafen im dienstrechtlichen Rahmen der Hitler-Jugend. Die Definition der Aufgabe der NSDAPParteigerichte durch den langjährigen Obersten Parteirichter Walter Buch als "Prüfung" dessen, "was nationalsozialistischer Ehrauffassung entspricht ${ }^{26}$, verdeutlicht, daß es sich um eine Scheidefunktion handelte.

Die Höchststrafe aller Disziplinar- und Strafordnungstexte bestand im Ausschluß aus der Organisation. Diese Strafkompetenz stand ausschließlich den $» \mathrm{HJ}-G e r i c h t e n « \mathrm{zu}$, in denen juristisch ausgebildete Jugendführer als $» \mathrm{HJ}-$ Richter in disziplinarischen Strafverfahren urteilten. Automatisch erstellten die Gerichte Warnkarten ${ }^{27}$. Die dauerhafte Entfernung, formal differenziert in ein »unehrenhaftes" dauerndes Ausscheiden und den Ausschluß, wurde als einziges Disziplinarmittel von mehreren Amnestien der Hitler-Jugend nicht erfaßt $^{28}$. Die organizistische Metaphorik dieser »schwersten « Strafe - im Parteirecht zur "Ausstoßung « von Fremdkörpern gesteiger ${ }^{29}$ - verrät bereits ihre Unumkehrbarkeit und ihre ausgrenzende Funktion. Quantitativ blieb die Anwendung der Strafe sehr begrenzt, weit weniger als $1 \%$ ihrer Mitglieder schied die Jugendorganisation auf disziplinarischem Wege aus - der Anteil scheint damit allerdings noch immer höher zu liegen als bei parteigerichtlichen Verfahren der NSDAP ${ }^{30}$. Die Disziplinierung der Masse erfolgte offensichtlich durch den Ausschluß weniger. Die Strafsanktion wurde dabei als äußerstes Mittel zum Schutz der Gemeinschaft begriffen, deren Schärfe weder durch häufigen Gebrauch, noch durch inkonsequente Folgen - etwa die Aufnahme in eine andere Parteigliederung - abgeschwächt werden sollte. Schließlich durfte

\footnotetext{
${ }^{26}$ Walter BUCH, Des nationalsozialistischen Menschen Ehre und Ehrenschutz, München ${ }^{5} 1939$ (Sonderdruck, Der Parteirichter, hg. vom Obersten Parteigericht der NSDAP), S. 25.

${ }^{27}$ Zucht und Ehre. Mitteilungs- und Schulungsblatt für die Dienstvorgesetzten der HitlerJugend 1 (1940). Vertraulich! Nur zum inneren Dienstgebrauch, hg. von der Reichsjugendführung (RJF) der NSDAP, Berlin 26.4.1940, S. 8.

${ }^{28}$ Sogenannte »Groß-Deutschland-Amnestie« nach der Annexion Österreichs im Mai 1938 (Amtliches Nachrichtenblatt des Jugendführers des Deutschen Reiches und der Reichsjugendführung der NSDAP, VV/12, 20.5.1938) und zu Beginn des Krieges (Dienstvorschrift für die Hitler-Jugend im Kriege, in: RB 1/K, 28.9.1939, S. 1-16, S. 15).

29 Das Gaugericht. Ausarbeitung noch nicht endgültig. Daher nicht veröffentlichen!, in: BA Berlin, Sammlung Schumacher, Nr. 380 [o.P.].

${ }^{30}$ Bezogen auf die Ausschlüsse zwischen Juni 1939 und August 1941, Reichsbefehl Sonderdruck 35/41 K. Die Schwierigkeit, quantitative Aussagen zur Parteigerichtsbarkeit zu treffen, problematisiert Armin NOLZEN, Parteigerichtsbarkeit und Parteiausschlüsse in der NSDAP 1921-1945, in: Zeitschrift für Geschichtswissenschaft 48 (2000) S. 965-989; vgl. ebenfalls Hubert ROSER, Nationalsozialistische Beamte auf der Anklagebank. NS-Parteigerichtsbarkeit und öffentliche Verwaltung in Südwestdeutschland 1933-1945, in: Comelia RAUHKÜHNE, Michael RUCK (Hg.), Regionale Eliten zwischen Diktatur und Demokratie. Baden und Württemberg 1930-1952, München 1993, S. 125-149. Nils BLOCK, Die Parteigerichtsbarkeit der NSDAP, Frankfurt a. M. 2001, beschränkt sich im wesentlichen auf einen Nachvollzug der Thesen von Donald MCKALE, The Nazi Party Courts. Hitler's Management of Conflict in His Movement, 1921-1945, Lawrence/Kansas 1974.
} 
die Reichsjugendführung auch aus strategischen Gründen ihre Kompetenz der umfassenden "Jugenderziehung" nicht durch hohe Ausschlußziffern in Frage stellen. $\mathrm{Ob}$ die praktizierten Ausschlüsse grundsätzlich eine breite $\mathrm{Ab}$ schreckungswirkung erzielten, läßt sich nicht überprüfen. Ihre Wirkungslogik setzte entweder die Identifikation des betreffenden "Jugendgenossen « mit der Organisation oder die Einschüchterung durch ihr offizielles Auftreten voraus. Versuche, einen Ausschluß durch Einsprüche oder gar Interventionen aufzuheben, belegen diese Strafwirkung zumindest für Einzelfälle ${ }^{31}$.

Der Ausschluß markiert die Schnittstelle von Integration und Ausgrenzung. Auf die Ordnung der Hitler-Jugend bezogen, bildet er die Grenze zwischen korrigierender Disziplinargewalt und staatlicher Bestrafung ${ }^{32}$. Die Disziplinargewalt bewertet Individuen aufgrund ihrer gesamten Erscheinung in Hinblick auf die Disziplin. Die Ausschlußgründe der Hitler-Jugend zielten in diesem Sinn auf eine grundsätzliche `Phänomenologie` als BDM-Mädel, Pimpf oder Hitler-Junge. Delikte und Verhaltensweisen, die HJ-Richter mit Ausschluß sanktionierten, benannten so einerseits die Belastbarkeitsgrenze der HJErziehung, zum anderen umreißen sie einen Gegenentwurf des Erziehungsideals.

Die auf den Warnkarten vermerkten Begründungen bezeichneten Infragestellungen der Ordnungsvorstellungen des nationalsozialistischen Jugendverbandes und Störpotential für die zeitlich unmittelbar anschließenden und auf ähnlichen Disziplinarprinzipien aufgebauten Ordnungen von Reichsarbeitsdienst (RAD) und Partei, bei den Jungen auch der Wehrmacht: Interne Disziplinlosigkeiten und Dienstversäumnisse wie eine "Befehlsverweigerung " oder gar eine »Meuterei" gefährdeten die Aufrechterhaltung des Jugend-Dienstes ebenso wie finanzielle Veruntreuungen. Mit der Autorität einzelner Führer untergruben sie zugleich auch die der Partei. Homosexualität stellte das sexistische und prokreative Gesellschaftsbild in Frage und galt überdies als kriminogen. Eigentumsdelinquenz gefährdete die materielle Gesellschaftsordnung. Als »ehrenrühriges " Verhalten bedrohte jedes strafrechtliche Delikt das Prestige der Gliederung selbst, ihre Glaubwürdigkeit und Identifikationskraft; Vorbestrafte wurden daher nicht aufgenommen. Ebenso delegitimierend wirkte auch eine nicht angemessene "Haltung" der Angehörigen. So wurde z.B. der Ausschluß eines »Jungmädels« mit ihrer »nicht BDM entsprechenden Le-

${ }^{31}$ Beispiele bilden z.B. die Intervention eines Pfarrers für Friedrich Emil Johann B., Jg. 1918, laut "Warnkartei« am 11.1.1936 aufgrund von »unnationalsozialistische[m] Verhalten« aus der $\mathrm{HJ}$ ausgeschlossen, in den Akten der Geheimen Staatspolizei, Staatspolizeistelle Düsseldorf, Außendienststelle Essen. Hauptstaatsarchiv Düsseldorf, RW 58-25 673; oder der Einspruch des ausgeschlossenen Hitler-Jungen Fritz $\mathrm{H}$. an die HJ-Gebietsführung Hochland, 24.8.1938; Vorgang in: Staatsarchiv Augsburg, HJ-Bann Memmingen, Nr. 25.

${ }^{32}$ Techniken eines Disziplinarsystems bei FouCAULT, Surveiller et punir, S. 209f., in Abgrenzung zur Strafjustiz, ibid. S. $214 f$. 
bensweise« begründet ${ }^{33}$. Nur sehr marginal finden sich tatsächlich politische Delikte. Distanz zum Nationalsozialismus hält die Kartei als "politische Unzuverlässigkeit« fest, deren Bezeichnungen von einer diagnostizierten »NSHaltlosigkeit« bis zu »staatsfeindlichen Umtrieben « rangieren. Ein solches Verhalten konnte z.B. in der Entfernung eines Aushanges aus dem Schaukasten eines SS-Blattes bestehen, wie auf einer Warnkarte von 1937 außergewöhnlich konkret vermerkt ist. Als "staatsfeindliche« oder »bündische Umtriebe« und damit als strafrechtliches Verbrechen galt ebenso die Fortführung alter Traditionen der verbotenen Jugendgruppen.

Rassebiologische Gründe der »Rassen-« und Staatsangehörigkeit und psychischer "Erbgesundheit", die bereits der Mitgliedschaft entgegenstanden, ergänzten diese unerwünschten Verhaltensformen. Der ebenso quantitativ wie in der intensiven Überwachung prominente Ausschlußgrund männlicher Homosexualität, der ein Fünftel aller Fälle der Warnkartei ausmacht, exemplifiziert dabei gleichermaßen eine zielgerichtete und intern eingestandene politische Instrumentalisierung sittlicher Normen, hier gegen die pauschal der Homosexualität verdächtigten Gruppen der Bündischen Jugend ${ }^{34}$. Außerdem verrät sich hier deutlich die sozialrassistische Begründung des Ausschließens, die erfolge, um eine unterstellte "Infizierung" und Ausbreitung sexueller und sozialer Devianz zu verhindern ${ }^{35}$.

Tatsächlich orientierten sich - mit Ausnahme von Vergehen und Versäumnissen im Dienstbetrieb und der sittlich-moralischen Charakterurteile - zahlreiche Ausschlußgründe an traditionellen wie neuen, seit 1933 eingeführten Kriminaltatbeständen. Unter den wenigen Kapitalverbrechen, die Ausschlüsse begründeten, finden sich vier junge Frauen im Alter von 17 bis 23 Jahren, die wegen Kindestötung aus dem BDM entfernt wurden; das im Zuge der rassi-

${ }^{33}$ Diese und die folgenden Zitate BA Berlin, Bestand BDC, Hitler-Jugend-Warnkartei.

${ }^{34}$ Vgl. exemplarisch: Kriminalität und Gefährdung der Jugend. Lagebericht bis zum Stande vom 1.1.1941, hg. von Jugendführer des Deutschen Reiches. Bearbeitet von William Knopp. o.O. o.J. Ediert in: Amo KLÖNNE (Hg.), Jugendkriminalität und Jugendopposition im NSStaat. Ein sozialgeschichtliches Dokument, Münster 1981, S. 5-228, hier S. 90, 94, 96f., 113; zur Kriminalisierungsstrategie gegenüber Bündischen Gruppen und einzelnen Angehörigen vgl. z.B. Bernhard JELLONEK, Homosexuelle unter dem Hakenkreuz. Die Verfolgung von Homosexuellen im Dritten Reich, Paderbom 1990, S. 324f.; Stefan KROLLE, »Bündische Umtriebe «. Geschichte des Nerother Wandervogels vor und unter dem NS-Staat. Ein Jugendbund zwischen Konformität und Widerstand, Münster 1986, S. 117-125, S. 81, 53; Amo KLÖNNE, Jugendprotest und Jugendopposition. Von der HJ-Erziehung zum Cliquenwesen der Kriegszeit, in: Martin BROSZAT u.a. (Hg.), Bayern in der NS-Zeit, Bd. IV, Herrschaft und Gesellschaft im Konflikt, Teil C, München 1981, S. 527-620, S. 615. Weibliche Homosexualität hingegen wurde aufgrund der unterschiedlichen Beurteilung und dem ungleichen Status der Geschlechter im öffentlichen Leben als sozial ungefährlich eingeschätzt und im "Altreich" nicht strafrechtlich verfolgt. Vgl. Claudia SCHOPPMANN, Zur Situation lesbischer Frauen in der NS:Zeit, in: Günther GRAU (Hg.), Homosexualität in der NS-Zeit. Dokumente einer Diskriminierung und Verfolgung, Frankfurt a. M. 2004, S. 35-42, S. 35f.

${ }^{35}$ Kriminalität und Gefährdung der Jugend. Lagebericht 1941, S. 115. 
schen NS-Bevölkerungspolitik für »erbgesunde« und »arische« Frauen scharf überwachte Abtreibungsverbot ist mit 15 Fällen in der Kartei ebenfalls nur marginal vertreten. Sie speichert jedoch auch mehrere Ärzte und einen Drogisten, die beruflich gegen den $\$ 218$ des Strafgesetzbuches verstoßen hatten darunter den in der Reichsjugendführung tätigen Arzt Wilhelm Schaper wegen "gewerbsmäßiger Abtreibung «. Mit dieser Ausnahme warnte die Kartei hier vor ihrem erweiterten Personenkreis, »jugendgefährdenden « Nichtmitgliedern.

Mit einem Anteil von 13\% an allen Ausschlüssen der Warnkartei bilden rassische Gründe immerhin ihren viertgrößten Teil, der sich in der überwiegenden Mehrheit gegen »Nichtarier«, also Personen jüdischer Abstammung richtete. Wie auch gegenüber nichtdeutschen Staatsangehörigen warnte hier die Kartei im ursprünglichen Sinn vor der Aufnahme der Betreffenden, wie Vermerke auf den Karten zeigen. So verhinderte die Warnkartei im Juni 1937 z.B. die Aufnahme der Schwestern Gertraud und Else St., deren Vater sie zur Teilnahme in JM und BDM anzumelden versuchte ${ }^{36}$. Der in nur $2 \%$ aller $» r a s s i-$ schen Ausschlüsse" genannte Grund des Kontaktes mit Juden bewirkte hingegen disziplinarrechtliche Entfernungen »arischer" Jugendlicher, die gegen das 1935 im sogenannten "Blutschutzgesetz« kodifizierte Verbot von Liebesbeziehungen oder gegen das in Parteigerichtsentscheidungen bestätigte Verbot sozialer und wirtschaftlicher Beziehungen verstoßen hatten ${ }^{37}$, wie die 16jährige Gerda $\mathrm{H}$., die "trotz Verwarnung freundschaftl. Verkehr mit Juden« unterhalten hatte, oder durch die Konsultation eines jüdischen Arztes.

Die disziplinarische HJ-Gerichtsbarkeit, die sich zum Befremden des grundsätzlich kooperativen Reichsministeriums der Justiz als "zugleich eine [...] Strafgerichtsbarkeit« verstand ${ }^{38}$, entwickelte sich zu einer Konkurrenz des staatlichen Strafanspruchs gegenüber Jugendlichen. Ihre Disziplinarmaßnahmen und Ermittlungen empfahl die Hitler-Jugend schon 1936 den Jugendrichtern selbstbewußt als "wertvolles Material [...], da sie den Justizbehörden ein Bild von der HJ-mässigen Beurteilung des Mannes und seiner Tat vermitteln « ${ }^{39}$. Sofern ein Delikt vorrangig als Disziplinlosigkeit begriffen wurde,

\footnotetext{
${ }^{36}$ Angesichts der zahlreichen Paragraphen, die NS-Organisationen vor jüdischen Mitgliedern sschützen، soliten, verdeutlicht Beate Meyers Hinweis auf "Unterwanderungsphantasien« zu Recht eine tiefenpsychologische Dimension der Abgrenzung. Beate MEYER, "Jüdische Mischlinge «. Rassenpolitik und Verfolgungserfahrung 1933-1945, Hamburg 1999, S. 259.

37 "Gesetz zum Schutze des deutschen Blutes und der deutschen Ehre« (»Blutschutzgesetz«), 15.4.1935, §§1-2, RGBl. 1935, Teil I, S. 1146; Walter TETZLAFF, Die Parteigerichtsbarkeit, in: Jugend und Recht 9 (1935) H. 9, S. 201-204, S. 204.

${ }^{38}$ Schreiben des Leiters des Personalamtes der Reichsjugendführung, Heinz-Hugo John, an das Reichsministerium der Justiz, 14.3.1936. BA R 3001/alt R 22/1174, Bl. 286. Großes Fragezeichen am Aktenrand.

${ }^{39} \mathrm{Ibid}$.
} 
konnte die disziplinarische Ahndung Vorrang erhalten und, ab 1943, die justizielle sogar erübrigen ${ }^{40}$.

\section{Dysfunktionalität und Radikalisierung in der Kriegszeit}

Mit der gesetzlichen Regelung der Pflichtmitgliedschaft durch den Erlaß der "Jugenddienstverordnung " im März und dem Kriegsbeginn im September bildet das Jahr 1939 eine organisationsgeschichtliche und politische Zäsur für die Rekonstruktion des Disziplinarsystems. Hier ist eine strukturelle Wende in Erfolg und Effizienz der Ordnungskonzeption zu vermuten, denn die Ausdehnung der beständig wachsenden Organisation erwies sich in dieser Situation als problematisch. Die Wirksamkeit des Ausschlusses als Strafe war einerseits durch das Interesse des Jugendlichen an der Teilnahme in der Gemeinschaft der Hitler-Jugend begrenzt, der ein Ausschluß aufgrund von "Interesselosigkeit « entsprach. ${ }^{41}$ Andererseits aber schränkte sie das Interesse des Staates an der loyalen Teilnahme möglichst vieler Jugendlicher selbst ein. Denn ab Herbst 1939 wurden die knapp neun Millionen Mitglieder der Hitler-Jugend als eine Art Kriegshilfsorganisation herangezogen, die im Verlauf des Krieges auf lokaler Ebene zunehmend an Bedeutung für die "Heimatfront« gewann ${ }^{42}$. $\mathrm{Zu}$ diesem Zeitpunkt mußte eine Anwendung der Strafe Ausschluß verhindern, daß sie durch zielgerichtete Verfehlungen genutzt werden konnte, um sich der "Jugenddienstpflicht« und den anschließenden staatlichen Verpflichtungen durch Arbeits- und Wehrdienst zu entziehen. Definiert als »Ehrendienste am deutschen Volk«, setzten sie die "Würdigkeit« der Dienstleistenden voraus; wen eine NS-Organisation einmal als »ehrlos" identifiziert und ausgeschlossen hatte, hatte diese verloren ${ }^{43}$. Hier erzeugte der Ausschluß, entgegen dem

${ }^{40}$ Reichsjugendgerichtsgesetz (RJGG) 1943, §30, Abs. 2 (Absehen von Strafverfolgung), $\S 31$, Abs. 1 (Einstellung des Strafverfahrens), präzisiert in der Allgemeinen Verfügung des Reichministers der Justiz über Hitler-Jugend und Strafrechtspflege, 18.12.1943, in: Deutsche Justiz 11 (1943) S. 569f. Dazu Gerhard KLEMER, Hitler-Jugend und Justiz, in: Das junge Deutschland 38 (1944) H. 2, S. 27-34, S. 32; vgl. auch Jörg WoLFF: Hitlerjugend und Jugendgerichtsbarkeit 1933-1945, in: Vierteljahrshefte für Zeitgeschichte 33 (1985) S. 640667, S. 666.

${ }^{41}$ So die Begründungen in den Satzungen aus der Vereinszeit der Organisation: Satzung der Hitler-Jugend-Bewegung e.V., Plauen 1928/29; Die Hitler-Jugend. Richtlinien, hg. von Reichsleitung der HJ, Plauen 1930.

${ }^{42} \mathrm{Vgl}$. dazu die umfassende Darstellung der Hitler-Jugend in der Kriegszeit: Michael BUDDRUS, Totale Erziehung für den totalen Krieg. Hitlerjugend und nationalsozialistische Jugendpolitik, München 2003.

${ }^{43}$ Reichsarbeitsdienstgesetz, 26.6.1935, RGB1. 1935, Teil I, S. 769, §§1, 5; Wehrgesetz, 21.5.1935, ibid. S. $609, \S \S 1,13$. 
Disziplinarinteresse, zugleich Leerstellen des ordnenden Zugriffs. Eine Organisation, die seit 1933 unermüdlich eine möglichst vollständige Mobilisierung beschwor, an welcher sie nach Kriegsbeginn durch die Ausweitung ihrer Einsatzgebiete und zeitgleichen Personalmangel durch die starke Einberufung des hauptamtlichen Hitler-Jugend-Führerkorps zur Wehrmacht festzuhalten erst recht gezwungen war, mußte auf solche Lücken notwendig reagieren. Gerade die Ausweitung des Ordnungssystems über den Rahmen der Partei hinaus auf den gesamten Staat, wie es die "Jugenddienstpflicht« mit der Erfassung der sgesamten Jugend anstrebte, führte also zu eigenen Problemen. Der erfolgreiche Einbau der Disziplinarmacht Hitler-Jugend in das staatliche Ordnungsgefüge veränderte auch die Rahmenbedingungen für das Disziplinarsystem. Durch verschiedene Strategien der Ausweitung und Kooperation erwies es sich jedoch als eine flexible, dynamische Ordnungssetzung.

Im zweiten Kriegswinter einigte sich die Reichsjugendführung mit der Wehrmacht auf eine wehrfreudige Auslegung des Begriffs der "Jugenddienstunwürdigkeit«, derzufolge "zwar jeder, der wehrunwürdig ist, zugleich jugenddienstunwürdig sein müsse, daß aber umgekehrt nicht jeder Jugenddienstunwürdige zugleich wehrunwürdig zu sein braucht ${ }^{44}$. Diese pragmatische Aufhebung der zuvor bestehenden "Wehrunwürdigkeit" aus der HitlerJugend ausgeschlossener junger Männer wurde entwicklungspsychologisch begründet: Die Jugendgemeinschaft sei durch »unwürdige« Mitglieder stärker gefährdet als die Gemeinschaften von Reichsarbeitsdienst und Wehrmacht und daher stärker auf den Schutz durch die Entfernungsstrafe angewiesen ${ }^{45}$.

Parallel versuchte die HJ-Gerichtsbarkeit, ihre überwachenden Disziplinierungstechniken gegenüber der Strafe zu stärken. Dazu erweiterte sie erstens 1940 den Kanon ihrer Disziplinarmittel um das neue Mittel des »Jugenddienstarrestes «, der nun auch erhebliche Verstöße älterer Hitler-Jungen innerhalb der Organisation korrigieren sollte und damit Ausschlüsse auffangen konnte $^{46}$. Zudem formulierten die HJ-Gerichte die Entfernungsstrafe nun vor-

\footnotetext{
${ }^{44}$ Jugendführer des Deutschen Reiches, Möckel, an Vertreter der Wehrmacht beim Jugendführer des Deutschen Reiches, 11.11.1941. BA Berlin R 3001/alt R 22/1175, Bl. 489-492, Bl. 489.

${ }^{45}$ Exemplarische Argumentation bei Roland FREISLER, Schreiben an den Jugendführer des Deutschen Reiches, 6.1.1942. Ibid. B1. 495-497, B1. 495f.

${ }^{46}$ Einführung der Dienststrafe "Jugenddienstarrest « durch Erlaß des Jugendführers des Deutschen Reiches, 17.9.1940, in: Amtliches Nachrichtenblatt VIIV/14, 25.10.1940, S. 139-148; Vorgang im Reichsjustizministerium: BA Berlin R 3001/alt R 22/1174, Bl. 509-511; als erste Bilanz der HJ-Gerichtsbarkeit Walter TETZLAFF, Der Jugenddienstarrest im ersten Jahr, in: Das junge Deutschland 36 (1942) H. 3, S. 52-57, S. 52. Zur Dienststrafe und der zeitgleich eingeführten Jugendstrafe »Jugendarrest« vgl. auch Jörg WOLFF, Jugend und Strafrecht im Nationalsozialismus, in: Zeitschrift für neuere Rechtsgeschichte 13 (1991) S. 41-66; Christine DöRNER, Erziehung durch Strafe. Die Geschichte des Jugendstrafvollzugs von 1871-1945, Weinheim 1991 sowie Maria MEYER-HOGER, Der Jugendarrest. Entstehung und Weiterentwicklung einer Sanktion, Baden-Baden 1998.
} 
rangig als »dauerhafte Ausscheidungen ${ }^{47}$, die eine Bewährungsmöglichkeit potentiell erhielten. Darüber hinaus vereinbarte die Jugendführung ergänzend anschließende "Betreuungen« der ausgeschlossenen Jugendlichen durch Jugendhilfe und Fürsorgeerziehung, die Kontrollücken schließen und eine mögliche Attraktivität des Ausschlusses verhindern sollten ${ }^{48}$. Trotz dieser Ausweitung der disziplinierenden Maßnahmen und einer Rhetorik, die eine zurückhaltende Verhängung des Ausschlusses beschwört, läßt sich jedoch keine Verringerung der praktizierten Ausschlußfälle nachweisen. Vereinzelte Zahlenangaben für das Jahr 1943 verweisen im Gegenteil sogar auf eine massiv erhöhte Straftätigkeit von annähernd 3000 Fällen im $\mathrm{Jahr}^{49}$.

Neue, kriegsbedingte Begründungen von Ausschlüssen verraten hingegen einen noch erhöhten Strafanspruch der Jugendführung, vor allem aufgrund von Mängeln in der persönlichen "Haltung «. Als Konsequenz justizieller Jugendstrafen erfolgten Ausschlüsse offenbar primär wegen Eigentumsdelinquenz oder »kriegsbedingter Straftaten wie Arbeitsvertragsbruch und Umgang mit Kriegsgefangenen «, wie aus einem Erfahrungsbericht über weiblichen Jugendstrafvollzug von 1943 hervorgeht $^{50}$. Im Zuge des Vorgehens gegen vermeintliche "Bummelanten « und "Arbeitsverweigerer" galt auch das eigenmächtige Verlassen des Arbeitsplatzes als Verstoß gegen die kriegswichtige Arbeitsdisziplin jetzt als Nachweis der »Unwürdigkeit ${ }^{51}$. Internierungen in den beiden 1940 und 1942 eingerichteten polizeilichen »Jugendschutzlagern" Moringen und Uckermark, die auch auf Vorschlag der Hitler-Jugend erfolgten, zogen

${ }^{47}$ Von den 2698 im Reichsbefehl Sonderdruck 35/41 K dokumentierten Entfernungen zwischen Juli 1939 und August 1941 wurden mit 714 Fällen nur 26,5\% als Ausschlüsse verhängt.

${ }^{48}$ Besprechung zur Jugendbetreuung, 22.12.1939. BA Berlin R 3001/alt R 22/1189, B1. 27. Zur "Jugendkontrollpolitik" im organisatorischen Rahmen der 1941 auf Initiative und unter Federführung der Hitler-Jugend eingerichteten »Reichsarbeitsgemeinschaft für Jugendbetreuung “ Alfons KENKMANN, Wilde Jugend. Lebenswelt großstädtischer Jugendlicher zwischen Weltwirtschaftskrise, Nationalsozialismus und Währungsreform, Essen 1996; Frank KEBBEDIES, Außer Kontrolle. Jugendkriminalitätspolitik in der NS-Zeit und der frühen Nachkriegszeit, Essen 2000 sowie neuerdings auch Michael BUDDRUS, Totale Erziehung, München 2003, S. 422-461.

49 Walter TETZlafF, Das Disziplinarrecht der Hitlerjugend. Entwicklung, gegenwärtiger Stand, Ausgestaltung, Berlin 1944 (Schriften zum Jugendrecht, 5), S. 46. Diese rein quantitativen Angaben erlauben jedoch weder eine Überprüfung noch eine Differenzierung nach den zugrundeliegenden Delikten; sie beruhen vermutlich, wie der überlieferte Reichsbefehl Sonderdruck 35/41 K, auf zusammenfassenden Meldungen der regionalen $\mathrm{HJ}$-Gerichte an die HJ-Gerichtsbarkeit der Reichsjugendführung.

${ }^{50}$ Bericht des Generalstaatsanwaltes Frankfurt a. M., Wackermann, 17.4.1943, BA Berlin R 3001/alt R 22/1305, Bl. 342.

${ }^{51}$ Entscheidung des HJ-Obergerichts, in: Der HJ-Richter, F. 5, Februar 1942, S. 8; zur Verfolgung der sogenannten »Asozialen im Betrieb« und der Einrichtung von speziellen »Arbeitserziehungslagern« vgl. Wolfgang AYASS, »Asoziale« im Nationalsozialismus, Stuttgart 1995, S. 177f.; Gabriele LOFTI, KZ der Gestapo. Arbeitserziehungslager im Dritten Reich, München 2000. 
den Ausschluß automatisch nach sich, da die in diesen Jugend-KZs ohne gerichtliche Verfahren oder Kontrolle inhaftierten Jugendlichen grundsätzlich als nicht mehr "gemeinschafts- « und besserungsfähig galten ${ }^{52}$. Die relative Deliktverteilung blieb dabei in der Kriegszeit gleich. Auffällig ist jedoch eine Verdoppelung des Anteils weiblicher "Warnfälle« (von 13\% auf 26\%), ein Trend, der sich offenbar weiter fortsetzte und sich nicht nur auf einen erhöhten Strafbedarf gegenüber den Frauen durch häufigere Auffälligkeiten zurückführen läßt. Mit den älteren männlichen Hitler-Jungen und HJ-Führern war auch die grundsätzlich kriminell aktivste Bevölkerungsgruppe als RAD- und Wehrmachtsangehörige der HJ-Gerichtsbarkeit entzogen. Das Verhalten der älteren Mädchen stand seit Kriegsbeginn zudem unter erhöhter Aufmerksamkeit der Überwachungsorgane, die massenhafte "Verwahrlosungs- und Entartungserscheinungen«, vor allem »in geschlechtlicher Hinsicht«, beobachteten und damit einen klassischen Topos im Umgang mit weiblicher Devianz neu belebten und mit einer »staatsgefährdenden « Bedeutung aufluden ${ }^{53}$. Denkbar

52 "Besserung« und daher Entlassung erwartete die Reichsjugendführung nur »ausnahmeweise« als Spätentwicklung des Jugendlichen. Schreiben Möckels, 11.11.1941. BA Berlin R 3001/alt R 22/1175, Bl. 489-492, B1. 491f.; Erlasse über die Einweisung Jugendlicher in polizeiliche Schutzlager des Reichsführers SS und Chefs der Deutschen Polizei, 25.4.1944, des Reichsministers des Inneren, 26.4.1944, und der Justiz, 27.4.1944 sowie des Jugendführers des Deutschen Reiches, 9.5.1944, in: Amtliches Nachrichtenblatt XII/8, 7.6.1944, S. 144-153; Detlev PEUKERT, Arbeitslager und Jugend-KZ. Die "Behandlung Gemeinschaftsfremder« im Dritten Reich, in: DERS., Jürgen REULECKE (Hg.), Die Reihen fast geschlossen. Beiträge zur Geschichte des Alltags unter dem Nationalsozialismus, Wuppertal 1981, S. 413-434, S. 417 u. 422; vgl. die Lebensgeschichte des bei Inhaftierung in Moringen aus der HJ ausgeschlossenen Erwin Rehn (Jg. 1927): Marie-Elisabeth REHN, Heider gottsleider. Kleinstadtleben unter dem Hakenkreuz. Eine Biographie, Basel 1992. Die Einweisung erfolgte jedoch nicht als »Dienststrafe der Hitler-Jugend«, wie Matthias von HELLFELD, Davongekommen! Erwachsenwerden im Holocaust, Frankfurt a. M. 1990, S. 28, behauptet; vgl. zu den »Jugendschutzlagern « Heinrich MUTH, Jugendopposition im Dritten Reich, in: Vierteljahrshefte für Zeitgeschichte 30 (1982) H. 3, S. 369-417; DERS., Das »Jugendschutzlager« Moringen, in: Dachauer Hefte 5 (1989) H. 5, S. 223-252; Katja LIMBÄCHER u.a. (Hg.), Das Mädchenkonzentrationslager Uckermark. Beiträge zur Geschichte und Gegenwart, Münster 2000, sowie Manuela NEUGEBAUER, Der Weg in das Jugendschutzlager Moringen. Eine entwicklungspolitische Analyse nationalsozialistischer Jugendpolitik, Mönchengladbach 1997.

53 Bericht über "Verwahrlosung weiblicher Jugendlicher«, im Januar 1941 vom Chef des Sicherheitsdienstes und des SD, an das Oberkommando der Wehrmacht und wverschiedene Reichsbehörden«, Institut für Zeitgeschichte, München, Fa 298, Bl. 212-254; vgl. in diesem Zusammenhang auch den Erlaß des »Reichsführers SS « zum "Schutz der weiblichen Jugend" vom 6.4.1942, BA Berlin, NS 7/181, Bl. 19, sowie Heike SCHMIDT, Gefährliche und gefährdete Mädchen. Weibliche Devianz und die Anfänge der Zwangs- und Fürsorgeerziehung, Opladen 2002, S. 284 u. 288; Kerstin KOHTZ, „Ich war ihm zu Willen, trotzdem sträubte ich mich«. Zur Sexualität »verwahrloster« Mädchen in der Weimarer Republik, in: Christina BEnNinghaus, DiES. (Hg.), „Sag mir, wo die Mädchen sind...«. Beiträge zur Geschlechtergeschichte der Jugend, Köln 1999, S. 169-191; und zum Topos in beiden Weltkriegen Birthe KUNDRUS, Kriegerfrauen. Familienpolitik und Geschlechterverhältnisse im Ersten und Zweiten Weltkrieg. Hamburg 1995, S. 212-220, S. 374-393. 
ist auch, daß angesichts des gravierenden »Führermangels« der Hitler-Jugend junge Männer gezielt zurückhaltender ausgeschlossen wurden.

Eine bedeutende qualitative Veränderung des Disziplinarwesens der HitlerJugend bedeutete jedoch die Partizipation an der wirkungsmächtigen, präventiv überwachenden »Jugendbetreuung « polizeilicher Organe. Damit bestimmte die Hitler-Jugend die massive, hypertrophe Jugendkontrollpolitik der Kriegsjahre maßgeblich mit und setzte sie in enger Zusammenarbeit mit Polizei und Justiz um: Der HJ-Streifendienst kontrollierte nun die Einhaltung der 1940 erlassenen Polizeiverordnungen $» z u m$ Schutze der Jugend $\aleph^{54}$. Dadurch erhielt die Überwachungsarbeit des Jugendverbandes einen bedrohlicheren Charakter, denn seit 1939 engagierten sich die Kooperationspartner des HJ-Disziplinarwesens in eskalierenden sozialrassistischen Aktionen wie der erbbiologischen vorbeugenden Verbrechensbekämpfung und der »Asozialen«-Verfolgung. Die Grenzziehung der Jugendführung zwischen erziehbaren, gemeinschaftsfähigen HJ-Mitgliedern und den "gemeinschaftsfremden" Auszuschließenden formulierte exakt die Zielgruppe dieser Verfolgungsorgane.

Diese verstärkte Kooperationspolitik der Kriegsjahre zeigt sich auch an der stillen Technologie der Karteiführung: Das noch 1944 im Justizministerium realisierte Projekt einer "gerichtlichen Erziehungskartei« verzeichnete neben den Jugendstrafen auch die "großen« Disziplinarmittel der Hitler-Jugend und damit die Entfernungsstrafen ${ }^{55}$. Hitler-Jugend-Rechtspolitiker begründeten ihre Dokumentation mit der hohen Bedeutung für den »Werdegang « eines Jugendlichen, der namentlich durch einen Ausschluß charakterisiert werde ${ }^{56}$. Das separate Jugenddisziplinarregister, das aufgrund der gerade seitens der Parteiorganisationen stark nachgefragten Strafregisterauskünfte eingerichtet wurde, stieß sofort auf lebhaftes Interesse bei Jugendämtern, Ordnungs- und Kriminalpolizei, Sicherheitsdienst, Wehrmacht und Reichsarbeitsdienst; die Auswertung für die »kriminalbiologische« Forschung galt als selbstverständlich. Insgesamt bezeugt diese späte Kartei, die zu einem Zeitpunkt eingerichtet wurde, zu dem weitaus prestigeträchtigere nationalsozialistische Karteierfassungsprojekte wie die »deutsche Volkskartei« bereits stillgelegt worden waren $^{57}$, die Aufwertung des Disziplinarrechts und seiner Strafe gegenüber dem staatlichen Jugendstrafrecht.

\footnotetext{
${ }^{54}$ Polizeiverordnung zum Schutze der Jugend, 9.3.1940, RGBl. 1940, Teil I, S. $126 f$.

${ }^{55}$ Gerhard KLEMER, Jugendstrafrecht und Hitler-Jugend, Berlin 1941 [Diss. jur. 1940/41], S. 91-93, S. 104; Vorgang BA Berlin R 3001/alt R 22/1206.

${ }^{56}$ KLEMER, Jugendstrafrecht, S. 91. Der Gerichtsreferendar war langjähriger Mitarbeiter der Reichsjugendführung, ab 1943 Geschäftsführer der »Reichsarbeitsgemeinschaft für Jugendbetreuung«.

${ }^{57}$ Götz ALY, Karl Heinz ROTH, Die restlose Erfassung. Volkszählen, Identifizieren, Aussondern im Nationalsozialismus, Frankfurt a. M. 2000, S. 133.
} 
»Erziehen« und »Warnen« - Sozialisationsziel und Gesellschaftsbild

Wie das allerdings praktisch nicht mehr wirksam gewordene Projekt der $» E r-$ ziehungskartei « verweist auch das "Warnkarteisystem « zwischen den Parteiorganisationen auf eine wichtigere Funktion als die bloBe bürokratische Archivierung von Disziplinarfällen. Durch die formalisierten Informationsverbindungen der Jugendorganisation zur Partei und von dort aus zu weiteren Suborganisationen entfaltete die strafweise Entfernung von Jugendlichen und Jugendführern erst recht ihre Wirkung. Das dichotomische Ordnungskonzept der Normierung als »erziehbar « betrachteter Jugendlicher und der Bestrafung und Ausstoßung »Unerziehbarer« setzte sich so in der Kaderpolitik des Nationalsozialismus fort. Galten Hitler-Jugend und BDM einerseits als Ort der Rekrutierung von Führungsnachwuchs, sollte der Ausschluß umgekehrt einem weitgehenden »Karrieretod" gleichkommen. Die automatisierten Warnungen vor den Ausgeschlossenen setzten auf diese Weise den Ausschluß aus der »Jugendgemeinschaft" in einen Ausschluß aus der "Volksgemeinschaft" um. Fand Disziplinierung durch Überwachung und erzieherische »Ehrenstrafen« innerhalb der Gemeinschaft statt, bestand die Strafe in der Ausgrenzung aus dieser. Mit der aufeinander aufbauenden Folge der juristisch gleichgestellten "Ehrendienste" von Arbeits- und Wehrdienst, die wie das ausgeprägte Führersystem auch der Integration dienten, korrespondierte dabei eine Reihe von Ausgrenzungsmechanismen, die sich an die strafweise Entfernung aus der Jugendorganisation anschließen konnten.

Das erfolgreiche Absolvieren der Hitler-Jugend bildete so die Voraussetzung eines Lebenslaufes durch verschiedene Stationen nationalsozialistischer Sozialordnungen. Als Ziel formulierte die Reichsjugendführung daher die Ausbildung einer extrem geschlechtsspezifisch geprägten "zweite[n] Generation des Nationalsozialismus «, welche die Volksgemeinschaftsidee der ersten Generation bereits lebe ${ }^{58}$. Mehrjähriger erfolgreicher »Jugenddienst« galt jedoch nicht nur als Voraussetzung für die Parteiaufnahme, politische Karrieren und die Nachwuchsfunktionen der Gliederungen ${ }^{59}$; neue Berufsbilder für erprobte Jugendführerinnen und Jugendführer entstanden gerade auch im Diszi-

${ }^{58}$ Gerhart WeHNER, Die rechtliche Stellung der Hitler-Jugend. Dresden 1939 [Diss. jur.], S. 154.

${ }_{59}$ Anordnung des Reichsschatzmeisters, Franz Xaver Schwarz, 25.10.1935, bekannt gegeben in: Reichsbefehl 11/I, 20.3.1936, S. 219f.; Verfügung Schwarz', 28.8.1936, über ihre weng[e] und streng[e]« Auslegung, in: Reichsbefehl 34/I, 2.10.1936, S. 745; ab 1943 war eine Überweisung des Führerkorps in die Partei automatisch vorgesehen. Führerdienst der HJ. Überweisung in die NSDAP und ihre Gliederungen, hg. von RJF der NSDAP, Berlin, 27.9.1942, S. 3f. Dietrich ORLOW, The History of the Nazi Party. 1933-1945, Pittsburgh 1973, S. 425. 
plinarbereich, etwa als qualifizierter Nachwuchs für Sicherheitspolizei, SD und die weibliche Kriminalpolizei ${ }^{60}$.

Im Selbstverständnis der Jugendorganisation galt der Jugenddienst darüber hinaus auch als Treuebeleg gegenüber dem NS-Staat insgesamt. Erst durch das "ehrenvolle Erfüllen" seiner Dienstpflichten weise »der junge Volksgenosse" nach, daß er "gewillt und geeignet ist, in Treue dem deutschen Volk und Reich zu dienen ${ }^{61}$. Diese besondere Treueverpflichtung verlangte seit 1935 das »Reichsbürgergesetz" den Trägern des »Reichsbürgerrechtes« und damit der »vollen politischen Rechte « ab - im Gegensatz zu den bloß »Staatsangehörigen «, denen keinerlei Rechtsgarantie zugesprochen wurde ${ }^{62}$. Die HitlerJugend hoffte sogar, ihre Mitgliedschaft würde als Kriterium für die Verleihung dieses privilegierenden neuen Rechtsstatus anerkannt. Die Verknüpfung von Rechtsansprüchen an zu erbringende "Treue» ist dabei eine Konsequenz aus dem nationalsozialistischen Rechtsdenken, das Verbrechen als Bruch der Treueverpflichtung gegenüber der Gemeinschaft neu definierte und Strafen als Ausdruck des geminderten Ansehens etablieren wollte. In diesem "Ehrenrecht« sollte der Rechtsstatus des Einzelnen sein Verhältnis zur Gemeinschaft abbilden. Deutlich stellt die rassistische "Volksgemeinschaft» hier den Bezugsrahmen der Rechtsgemeinschaft ${ }^{63}$.

Damit behauptete sich das Disziplinarwesen des Jugendverbandes als ständige "Auslese Befähigter und der Ausmerzung Schädlicher « ${ }^{64}$ nicht nur für den eigenen Apparat, sondern für die gesamte "Volksgemeinschaft«. Wenn Schirach pädagogisierte, der Wert der Jugendorganisation bestehe für die Jugendlichen im Einüben und im Verständnis von Disziplin ${ }^{65}$, läßt sich neben der hier gemeinten Charakterübung auch der Hintergrund des Disziplinarkon-

${ }^{60}$ Günther KAUFMANN, Jugendführer - ein Beruf des 20. Jahrhunderts, in: Wille und Macht. Halbmonatsschrift des jungen Deutschland. Zentralorgan der nationalsozialistischen Jugend 6 (1938) H. 5, S. 31-33; Reichsbefehl 23/43 K, 26.8.1943, S. 404f;; William KNOPP: Das Überwachungswesen der Hitler-Jugend, in: Das junge Deutschland 38 (1944) H. 5/6, S. 97107, S. 106; Anordnung des Personalamtes, in: Rundschreiben der Reichsjugendführung, F. 16/44, 17.8.1944, S. 341 .

${ }^{61}$ ANONYM, Die Jugenddienstpflicht, in: Das junge Deutschland 36 (1942) H. 5, S. 91-100, S. 99.

${ }^{62}$ Reichsbürgergesetz, 15.9.1935, RGBl. 1935, Teil I, S. 1146. Vgl. dazu Comelia ESSNER, Die »Nürnberger Gesetze« oder die Verwaltung des Rassenwahns, 1933-1945, Paderborn 2002; zur Fragmentierung und Auflösung der Staatsbürgerschaft im Nationalsozialismus auch Dieter GOSEWINKEL, Einbürgem und Ausschließen. Die Nationalisierung der Staatsangehörigkeit vom Deutschen Bund bis zur Bundesrepublik Deutschland, Göttingen ${ }^{2} 2003$, S. 369-420, zum Reichsbürgergesetz S. 383-393.

${ }^{63}$ Zur Dynamik dieser staatsrechtlichen Verschiebung Michael WILDT, Die politische Ordnung der Volksgemeinschaft. Emst Fraenkels „Doppelstaat" neu betrachtet, in: Mittelweg 36, 12 (2003) H. 2, S. 45-61.

${ }^{64}$ Hans-Helmut DIETZE, Die Rechtsgestalt der Hitler-Jugend. Eine verfassungsrechtliche Studie. Berlin 1939, S. 113.

${ }^{65}$ SCHIRACH, Hitler-Jugend, S. 69. 
zeptes herauslesen: Aus Sicht der NS-Organisation bestand der biographische `Gewinn ‘ür die Jugendlichen in der geglückten Disziplinierung, die eine Normierung und Normalisierung im Hinblick auf die nationalsozialistische Gesellschaftsordnung bedeutete und darüber hinaus spezifische Karrieremöglichkeiten des Regimes im Rahmen des Parteigefüges und der hier vergrößerten sozialen Mobilität eröffnete. Der Hitler-Jugend-Dienst diente so als Probe auf die gewünschte "Gemeinschaftsfähigkeit«, da er eine "neue Möglichkeit der Wesenserkenntnis Jugendlicher auf Grund des Verhaltens in der Jugendgemeinschaft « zu bieten schien ${ }^{66}$. Dieses eingestandene personelle »Auslesesystem " in der Jugendorganisation funktionierte in zwei Richtungen: Einerseits fand Auslese »im negativen Sinne« als »Abstoßung alles Unbrauchbaren und Untauglichen" statt, andererseits »im positiven Sinne«, als Suche und Förderung geeigneten Führernachwuchses ${ }^{67}$. Diese funktionelle Dichotomie entspricht den sich ergänzenden Polen von Gratifikation und Sanktion des Disziplinierens ${ }^{68}$. Strafen bildete auch im Disziplinarsystem der Hitler-Jugend lediglich die unverzichtbare Kehrseite eines doppelseitigen Mechanismus.

Dem unmittelbaren Zweck der Warnkartei folgend blieben den ausgeschlossenen Mädchen und Jungen die Partei und ihre Gliederungen in der Regel verschlossen, ebenso Führungsstellungen, NS-Schulen und, auch nach 1941, Offizierskarrieren in der Wehrmacht. Der HJ-Richter Walter Tetzlaff begriff den Ausschluß daher als "politisches Todesurteil ${ }^{69}$. Restriktionen betrafen auch den Zugang zum öffentlichen Dienst, da 1935 eine mehrjährige erfolgreiche Teilnahme im Jugendverband als Bewerbungsvoraussetzung festgeschrieben wurde $^{70}$. Gerade in den ersten Jahren des Nationalsozialismus ermöglichte die Mitgliedschaft auch den Zugang zu begehrten Arbeitsplätzen und vor allem zu Lehrstellen, da auch Privatunternehmen in Industrie und Handwerk, teilweise auf Anweisung staatlicher Stellen, einen Mitgliedschaftsnachweis verlang$\operatorname{ten}^{71}$. Seit der Eingliederung der "Jugend der DAF« in die Hitler-Jugend Ende 1933 drohte Arbeitsplatzverlust, da die notwendige Mitgliedschaft im NSBerufstätigenverband ebenfalls die Teilnahme in der Jugendorganisation vor-

${ }^{66}$ KLEMER, Jugendstrafrecht, S. 101.

${ }^{67}$ ANONYM, Disziplinarordnung, S. $41 \mathrm{f}$.

${ }^{68}$ FouCAULT, Surveiller et punir, S. 212.

${ }^{69}$ Walter TETZLAFF, Nationalsozialistische Rechtsphilosophie, in: Der HJ-Richter, F. 4, Juni 1941, S. 3-7, S. 6. Einzelfälle aus dem Bestand der Personalunterlagen im BA Berlin, BDC, dokumentieren jedoch Ausnahmen, bei denen andere, glaubwürdig bezeugte ,Verdienste ‘ für die »Bewegung « die Jugendverfehlung ausgleichen konnten.

${ }^{70}$ Runderlaß des Reichs- und Preußischen Ministeriums des Inneren, 1.11.1935, bekannt gegeben in: Reichsbefehl 8/I, 28.2.1936.

${ }_{11}$ Politischer Lagebericht des Oberpräsidenten der Rheinprovinz, 16./17.8.1935, in: Bernhard VOLLMER (Hg.), Volksopposition und Polizeistaat, 24, Stuttgart 1957, S. 267-274, S. 274; weitere Beispiele bei KENKMANN, Wilde Jugend, S. 70. 
aussetzte $^{72}$. Dieses indirekte Arbeits- und Lehrstellenverbot für Ausgeschlossene und Nichtmitglieder wirkte zunächst existenziell bedrohlich; die Verweigerung einer Ausbildungsmöglichkeit und die damit verbundene Unterqualifizierung und langfristige Minderung des Lebensstandards bot aber auch nach Erreichen der Vollbeschäftigung weiterhin Einschüchterungspotential. Auch im Bildungsbereich wurde die "nationale Zuverlässigkeit» anhand der Mitgliedschaft geprüft ${ }^{73}$. Zeitzeugen erinnern sich auch an die Drohung, Nichtmitglieder würden nicht zur Abiturprüfung zugelassen ${ }^{74}$. Planungen der $\mathrm{HJ}$ Gerichtsbarkeit, die Schulen "mit dem Ziel der gleichzeitigen Verweisung von der Höheren Schule« in das disziplinarische Warnsystem einzubeziehen, hätten für Ausgeschlossene eine höhere Schulbildung und den Zugang zu sämtlichen akademischen Berufen grundsätzlich verhindert ${ }^{75}$. Weitaus schwerwiegendere Folgen konnte die Entfernung jedoch nach sich ziehen, wenn eine weitere Auffälligkeit andere Ausgrenzungsmechanismen einleitete. In nur zum Teil realisierten Plänen der Reichsjugendführung zeichnete sich eine snegativer Laufbahn repressiver Bewahrungsstationen von Fürsorgeerziehung und "polizeilichen Jugendschutzlagern« für ausgeschlossene Jugendliche $a b^{76}$. Seit 1940 schlossen sich tatsächlich KZ-Einweisungen an die mit der Volljährigkeit des Zöglings endende Fürsorgebewahrung an. Die Stigmatisierung durch die organisationsinterne Warnung erwies sich hier als direkte Vorstufe der Verfolgung vermeintlich »Gemeinschaftsfremder«. In den überwiegenden Fällen, in denen der Ausschluß aufgrund eines Kriminaltatbestandes erfolgte, bedeutete der Warneintrag durch das polizeiliche Meldesystem zugleich Straf-

\footnotetext{
${ }^{72}$ Vereinbarung zwischen dem Führer der Deutschen Arbeitsfront (DAF), Robert Ley, und dem Reichsjugendführer, Schirach, 8.12.1933, in: JAHNKE, BUDDRUS (Hg.), Deutsche Jugend, 24, S. 81f;; Erlaß der Leiter des Organisations- und des Jugendamtes der DAF, in: Heinrich RoTH, Katholische Jugend in der NS-Zeit unter besonderer Berücksichtigung des Katholischen Jungmännerverbandes. Daten und Dokumente, Düsseldorf, Nr. 46, S, 104; Heinz BOBERACH, Jugend unter Hitler, Düsseldorf 1982, S. $26 f$.

73 Verordnung über die zahlenmäßige Begrenzung des Zugangs zu den Hochschulen, 9.1.1934, auf Grundlage des Gesetzes gegen die Überfüllung deutscher Schulen und Hochschulen, 25.4.1933, sogenannter »Abiturientenerlaß«, RGB1. 1933, Teil I, S. 225f.; Barbara SCHELLENBERGER, Katholische Jugend und Drittes Reich. Eine Geschichte des Katholischen Jungmännerverbandes 1933-1939 unter besonderer Berücksichtigung der Rheinprovinz, Mainz 1975, S. 47.

${ }^{74}$ Zitiert bei MEYER, »Jüdische Mischlinge«, S. 256.

${ }^{75}$ Entwürfe zum Disziplinarrecht der Hitler-Jugend, S. 3. Durchführungsverordnung zum Gesetz über die Hitler-Jugend, in: TETzLAFF, Disziplinarrecht, S. 150-158, S. 152. REMPEL, Hitler's children, S. 69 , beurteilt dies irrtümlich als Realität.

${ }^{76}$ Günter KaufmanN, Das kommende Deutschland. Die Erziehung der Jugend im Reich Adolf Hitlers, Berlin ${ }^{3} 1943$, S. 326.
} 
verfolgung, wobei zahlreiche Sonderverordnungen den strafrechtlichen Schutzraum Jugendlicher nach 1939 erheblich einschränkten ${ }^{77}$.

Die Warnung vor Ausgeschlossenen schloß diese damit einerseits von Förderung und Karriere aus und beraubte sie andererseits grundlegender Rechtsansprüche. Der Idee des Warnens lag dabei das biologistisch-sozialrassistische Konzept zugrunde, das eine "Verseuchung guten Menschenmaterials" durch den Einfluß »verdorbener Elemente« verhindern zu müssen glaubte. Das gesamte prüfende Disziplinarsystem übernahm das rassenhygienische Instrumentarium aus Maßnahmen zur positiven "Auslese" und negativen "Ausmerze«. Die Jugenddisziplinierung verfolgte dabei langfristige Effizienz, da »die Reinhaltung und Ertüchtigung der Jugend [...] auf die Dauer die Säuberung des gesamten Volkskörpers « bedeute, wie der spätere Reichsjugendführer Artur Axmann formulierte ${ }^{78}$. Über den Begriff der »Ehre« wurden Rechte und Pflichten im Staat an die rassistisch definierte Gemeinschaft geknüpft. Den ausgegrenzten Jugendlichen wurde überdies der Anspruch auf eine - in der Hitler-Jugend freilich zur Sozialisation reduzierte - "Erziehung" und eine dem modernen Jugendbegriff entsprechende Jugendzeit als Moratorium zur Persönlichkeitsentwicklung und -reifung ${ }^{79}$ ebenso abgesprochen wie die durch die Staatsangehörigkeit gewährten Rechte und Schutzräume. Die disziplinierte »Volksgemeinschaft « basierte auf dieser Abgrenzung nach innen ebenso wie auf den Integrationsangeboten und der nationalen Abgrenzung nach außen. »HJ-Fähigkeit" funktionierte so als Selektionskriterium für die "Volksgemeinschaft«, die durch die Disziplinarstrafe des Ausschlusses umgesetzt wurde. Nach einer ethnisch-rassistischen Vorauswahl durch die Aufnahmekriterien bezeichneten die Ausschlußbegründungen den sozialen Rassismus nach innen gegen »Störer der Gemeinschaftsordnung ${ }^{80}$. Das akribisch organisierte Warnsystem selbst ist ein direkter Ausdruck dieser Orientierung. Der Massenzugriff des nationalsozialistischen Erfassungswesens, das in der kompakten Warnkartei als Komplement die Mitgliederverwaltung ergänzte, erweist sich darin als voraussetzungsvolle Technik. Auch wenn dieses Karteisystem gegenüber ausgefeilten sozialstatistischen Untersuchungs- und Erfassungsprojekten im Nationalsozialismus wie der »Volks-« oder der »Volkstumskartei« vergleichsweise harmlos wirkt, versteckt sich auch hinter seiner Einfachheit eine "Vision der gereinig-

" „Verordnung zum Schutz gegen jugendliche Schwerverbrecher«, 4.10.1939, RGB1. 1939, Teil I, S. 2000, \$1; Verordnungen wüber die unbestimmte Verurteilung Jugendlicher«, 20.9.1941, RGB1. 1941, Teil I, S. 567, §1; 6.1.1942; RGB1. 1942, Teil I, S. 18, §1.

${ }_{78}$ Artur AXMANN, Zum Jugendstrafrecht, in: Das junge Deutschland 33, 3 (1939) S. 97.

${ }^{79} \mathrm{Vgl}$. dazu Winfried SPEITKAMP, Jugend in der Neuzeit. Deutschland vom 16. bis zum 20. Jahrhundert, Göttingen 1998, S. $292 \mathrm{f}$.

${ }^{80}$ KLEMER, Jugendstrafrecht, S. 49. 
ten Gesellschaft ${ }^{81}$. Die Disziplinarpolitik der Hitler-Jugend stellt damit den zentralen Zugriff der NS-Rassenpolitik auf Kinder und Jugendliche dar. Die eigentlich paradoxe Figur des Ausschlusses aus einer auf möglichst vollständige Erfassung zielenden Organisation spiegelt in ihrer Funktionalität und Dynamik die Herrschaftstechnik der »Volksgemeinschaft«. Ihre Funktionsfähigkeit als Zusammenspiel von Integration und Ausgrenzung erforderte eine deutliche Bezugsgrenze. Die Hitler-Jugend beschrieb diese Grenze analog zu ihrer Aufgabe einer nationalsozialistischen politischen und "sittlichen « Jugenderziehung als "Erziehungsfähigkeit« und markierte die Betroffenen durch ihre Disziplinarstrafe. Die Warnkartei wies diese Stigmatisierung bei Bedarf nach, um diese biographisch erste soziale Ausgrenzung aus der " Jugendgemeinschaft« systematisch fortzusetzen. Insofern erweist sich diese »ungeheuer primitive« Erfassung ${ }^{82}$ der im Nationalsozialismus omnipräsenten Karteiarchive als kennzeichnend für die Herrschaftstechnik, die bürokratische Effizienz und Flexibilität mit normativ-ideologischer Orientierung verband.

Für das Verhältnis der Jugendorganisation zum nationalsozialistischen Staat ergibt sich dabei ein widersprüchlicher Befund. Als doppelte Institution auf typische Weise in die Verschränkung von Parteiorganisation und Staatsbehörden eingebaut, diente die Hitler-Jugend sowohl strukturell wie mit ihrer Funktion der politischen Sozialisation der Jugendlichen einer nationalsozialistischen Durchdringung der Gesellschaft. Über die Mitgliedschaft definierte sie die gesellschaftliche Gruppe der »deutschen Jugend«, die als künftige Kader und Angehörige die „Volksgemeinschaft“ garantieren sollten. In Übereinstimmung mit der Parteiorganisation trat die Jugendführung hier unverhüllt als gesellschaftlicher Konstrukteur auf. Die Disziplinarfunktion der Hitler-Jugend unterstützte eine gesellschaftliche Auslese für die gesamte Gesellschaft und für künftige Funktionsträger und -eliten in Partei und Staat. Dabei beruhte die Legitimation eines eigenen Dienststrafrechtes gleichermaßen auf der Aufwertung der Organisation durch einen staatlichen Geltungsanspruch wie auf der ideologischen Bindung an die Partei. Der Dualismus der Jugendführung öffnete hierbei eine wirksame "Zwischenzone von innerorganisatorischer Bedeutung und öffentlichem Recht ${ }^{83}$. Der zielstrebige Ausbau des Disziplinarwesens belegt auch den Ehrgeiz und die wechselvolle Entwicklungsdynamik der selbstbewußten Organisation. Er steht zugleich für eine Aufwertung von Disziplinar- und Standesrechten im juristischen Diskurs nach 1933. Neben der Imitation staatlicher Gerichtsbarkeit - in der Orientierung am strafrechtlichen Sanktionskatalog, der Tendenz zur Verrechtlichung, Bürokratisierung und to-

8) Patrick WAGNER, Volksgemeinschaft ohne Verbrecher. Konzeptionen und Praxis der Kriminalpolizei in der Zeit der Weimarer Republik und des Nationalsozialismus, Hamburg 1996, S. 405.

${ }^{82}$ AYASS, "Asoziale«, S. 110.

${ }^{83}$ MUTH, Jugendopposition, S. 394. 
talen Erfassung - beanșpruchte die HJ-Gerichtsbarkeit auch eine konkurrierende Position gegenüber den staatlichen Institutionen. So wirkten die Disziplinarmaßnahmen und -institutionen teilweise als Ersatz für staatliche Strafmaßnahmen; die Bindung der Mitgliedschaft an das Reichsbürgergesetz erfolgte hingegen nicht. In der Kriegszeit fügten sich die Disziplinarpolitik und Disziplinarmechanismen der Jugendorganisation in enger Kooperation mit staatlichen Behörden und Exekutivinstanzen, vor allem der Polizei, und parteiamtlichen Institutionen schließlich in eine umfassende disziplinierende Jugendkontrollpolitik ein.

\section{Résumé français}

Cette contribution étudie une figure centrale de la dictature nazie, à savoir la technique de domination de la »communauté du peuple (Volksgemeinschaft). Cette conjonction de mécanismes d'intégration et d'exclusion est observée à partir de la politique disciplinaire de l'organisation de la »Jeunesse hitlérienne« (Hitler-Jugend, HJ). Cette organisation, qui relevait à la fois de l'État et du parti et réunissait tous les enfants et adolescents allemands nonjuifs entre 10 et 18 ans, développa et pratiqua les techniques disciplinaires de contrôle normalisateur, de la punition d'exclusion et du recensement documenté, dans le cadre d'un système réglementaire disciplinaire et pénal autonome.

Alors que, dans la $\mathrm{HJ}$ ou dans la »Ligue des jeunes filles allemandes« (Bund Deutscher Mädel, BDM), les infractions mineures commises contre la discipline étaient sanctionnés par les "punitions d'honneur« (Ehrenstrafen) qui étaient considérées comme »pédagogiques«, la peine maximale consistait en l'exclusion »déshonorante« de l'organisation. Les jeunes étaient renvoyés et exclus de l'organisation éducative de la $\mathrm{HJ}$ et par là même, symboliquement, de la société. L'aptitude à faire partie de la $\mathrm{HJ}$ fonctionnait comme un critère de sélection, la sanction disciplinaire y fut le moyen. Ainsi on décidait aussi, dans cette organisation authentiquement national-socialiste, de l'appartenance à la »communauté du peuple«, de la participation à la société national-socialiste, des chances d'accès à un emploi ou d'évolution de carrière, du statut juridique ou de la sécurité juridique des individus. La figure paradoxale de la peine maximale est au centre de cette analyse. On a utilisé comme source principale le "fichier d'avertissement" (Warnkartei) de la $\mathrm{HJ}$, dans lequel les fonctionnaires et les collaborateurs des organisations de jeunesse enregistraient les exclusions de jeunes membres pour empêcher une éventuelle réintégration. Les motifs d'exclusion indiquaient les questions que l'organisation de jeunesse se posait dans le domaine de sa représentation de l'ordre ainsi que sur le potentiel de perturbation éventuel des organisations pour les adultes comme le »Reichsarbeitsdienst« (RAD), la Wehrmacht et la NSDAP, dont l'ordre était basé sur des principes disciplinaires similaires.

La conception d'un ordre dichotomique faisant la distinction entre la normalisation de jeunes considérés comme »éducables« et la punition et l'exclusion de jeunes »nonéducables« se poursuivait dans la politique d'encadrement et de persécution du nationalsocialisme. La HJ faisait ainsi office, d'une part, de lieu de mobilisation et d'intégration, dans la mesure où elle recrutait la relève du personnel d'encadrement pour une société national-socialiste et, d'autre part, d'instance qui - en accord avec la NSDAP - planifiait, prescrivait et mettait à exécution l'exclusion. En tant que processus pour établir l'ordre sociale avec de vastes conséquences sociales et politiques, le mécanisme disciplinaire double de la $\mathrm{HJ}$ met en lumière une mécanique de précision de la domination national-socialiste qui combinait une efficacité et flexibilité bureaucratique à une orientation idéologique normative. 